\title{
Noise Performance and Chopper Frequency in Integrated Micromachined Chopper-Detectors in Silicon
}

\author{
R. F. Wolffenbuttel and G. de Graaf
}

\begin{abstract}
The noise behavior of the basic transimpedance amplifier has been investigated in the frequency range in between $100 \mathrm{kHz}$ and $1 \mathrm{MHz}$. At frequencies below $10 \mathrm{kHz}$ JFETbased operational amplifiers are preferred, because of the low equivalent input current noise that dominates overall noise performance. At frequencies beyond $10 \mathrm{MHz}$ circuits with a bipolar input stage are generally used, because the equivalent input voltage dominates noise performance due to the capacitive source impedance of the photodiode at such frequencies. The transitional frequency range indicated has become important due to the increased operating frequency of optical choppers. It will be shown that a transimpedance amplifier with a bipolar input stage is preferred at intermediate frequencies and that the noise performance limits the operating frequency in an integrated micromechanical chopper rather than its inertia.
\end{abstract}

\section{INTRODUCTION}

C ONVENTIONAL photodiode applications in instrumentation are either in the sub-kiloHertz range for the detection of mechanically chopped light or at frequencies exceeding $10 \mathrm{MHz}$ when used for interfacing optical fibers. Detector noise is shot-noise limited in the former application, whereas the thermal noise in the series resistance dominates in optical telecommunication. Low frequency readout, therefore, benefits from the implementation of readout circuits with a small equivalent input noise current, whereas the equivalent noise voltage is of lesser importance due to the high value of the source impedance, thus favouring FET readout. Low-noise high-frequency readout, however, requires signal conditioning circuits with a predominantly equivalent noise voltage, which implies the implementation of bipolar transistors [1], [2] Basically, the optimum equivalent noise impedance of the readout becomes frequency dependent due to the parasitic junction capacitance and series resistance of the photodiode. So far, noise optimization at intermediate frequencies $(100$ $\mathrm{kHz}-1 \mathrm{MHz}$ ) has received little attention due to lack of applications. However, LED-photodetector distance measuring systems are usually operating at such frequencies. Moreover, advanced micromechanical fabrication techniques allow the realisation of mechanical choppers operating at much higher frequencies than those of conventional systems.

Manuscript received July 1, 1994; revised October 15, 1994.

The authors are with the Laboratory for Electronic Instrumentation, Department of Electrical Engineering, Delft University of Technology, Mekelweg 4 2628CD Delft, The Netherlands.

IEEE Log Number 9408715.

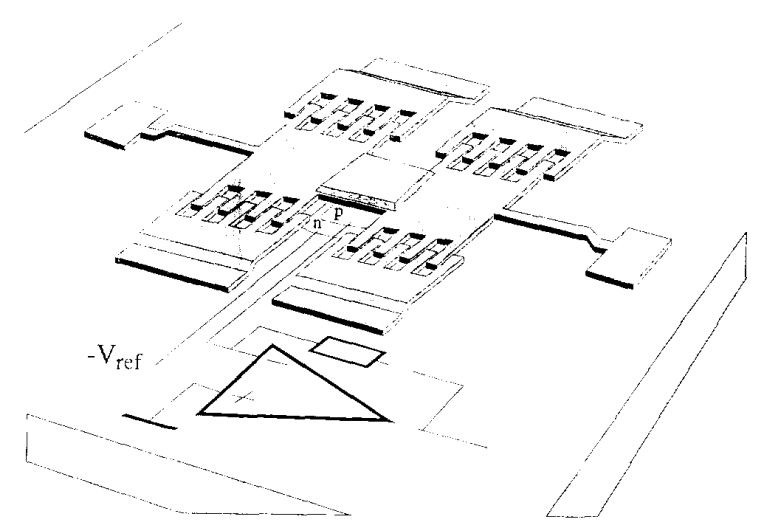

Fig. 1. Structure of the micromachined optical chopper.

Recent advances in silicon micromachining technologies enable the fabrication of single-chip optical chopper-detector systems with integrated readout circuits in silicon. Surface micromachining is one of these techniques and is based on the depostion and patterning of sacrificial and structural thin films on a silicon substrate in which integrated readout circuits are already fabricated [3]. First a phosphorous-doped glass sacrificial layer of about $0.5-2 \mu \mathrm{m}$ thickness is deposited and patterned to leave contacts where the subsequently deposited polysilicon structural layer of about the same thickness can be anchored to the substrate. The polysilicon can be patterned to form e.g., strips. Finally, the sacrificial layer is selectively removed by wet-etching in $\mathrm{HF}$, leaving free-standing polysilicon bridges. The micron-size of the thin-film chopper would allow operation at much higher frequencies compared to conventional choppers. A typical integrated chopper is composed of an electrostatically actuated comb drive [4], which displaces a reflecting film over the detector to give alternating transmission and blocking of impinging light onto the integrated photodiode as shown schematically in Fig. 1.

The major problem in such a device is the crosstalk between the drive circuits and the coherent detector, as the drive frequency and chopper frequency are coupled in case of synchronous operation. Moreover, a detailed analysis of the noise performance is required in order to set the upper limit to the operating frequency. This paper will show that, although the inertia of the chopper would allow operation up to very high frequencies limited only by squeezed-film damping, the 


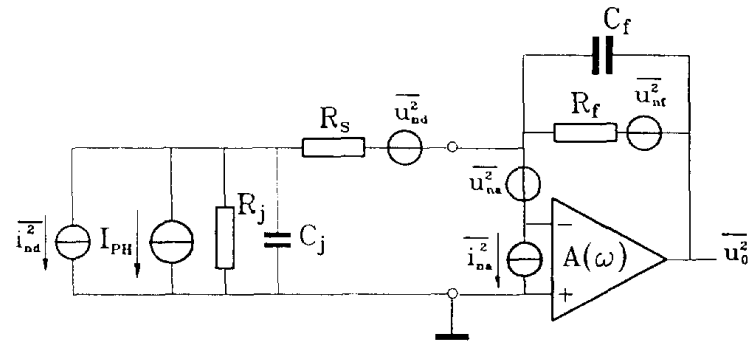

Fig. 2. Equivalent noise schematic of the photodiode readout.

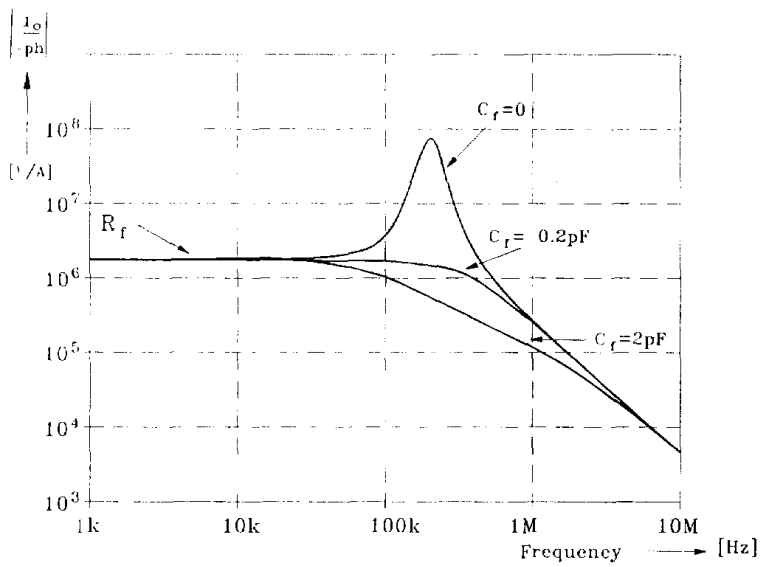

Fin. 3. Gain plot of the transimpedance amplifier

noise performance would limit the maximum frequency to about $100 \mathrm{kHz}$. Moreover, readout circuits based on bipolar input stages have better noise performance at such frequencies compared to FET-based input stages.

\section{Photodiode Readout}

The circuits conventionally applied for the readout of a photodiode is based on the transimpedance amplifier shown schematically in Fig. 2. The transimpedance is in principle equal to $R_{t}$ parallel to $C_{t}$, however when also considering diode parasitics and open-loop gain $A(\omega)=A_{o} /\left(1+j \omega \tau_{v}\right) \simeq$

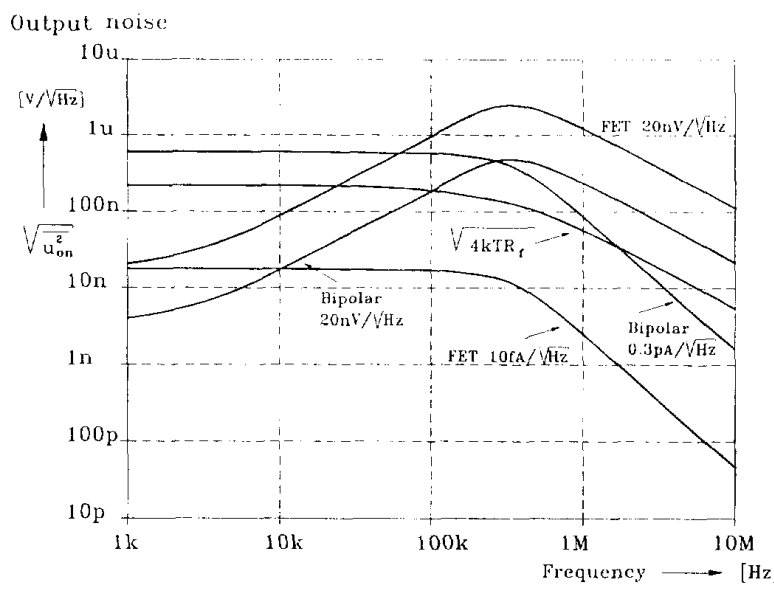

Fig. 4. Output noise resulting from the various noise source in the transimpedance amplifier for both bipolar and FET input stages.

$A_{o} / j \omega \tau_{v}$, it is more accurately expressed as

$$
\frac{u_{o}}{i_{p h}} \simeq \frac{R_{f}}{1+j \omega\left(R_{f} C_{f}+R_{s} C_{j}\right)-\omega^{2} R_{f} C_{j}\left(R_{s} C_{f}+\frac{\tau_{v}}{A_{o}}\right)}
$$

where $R_{s}$ denotes the series resistance, $C_{j}$ the junction capacitance, $R_{f}$ the feedback resistance, $C_{f}$ the feedback capacitance and $i_{p h}$ the photocurrent through the diode. The transimpedance amplifier is known for its peaking at the resonance frequency and the feedback capacitor $C_{f}$ should be tuned for critical damping as shown in Fig. 3. In this circuit $R_{s}=600 \Omega, R_{f}=2 M \Omega, C_{j}=50 \mathrm{pF}$, and $C_{f}=0.2 \mathrm{pF}$ have been used. For noise analysis the different noise sources of the circuit and photodetector are analysed by determining the equivalent output noise voltage (see (2), shown at the bottom of the page)

Fig. 4 shows the various equivalent input noise components for both an operational amplifier and a JFET device. The total output noise voltage has been calculated using SPICE with $A_{o}=100 \mathrm{~dB}$ and $\tau_{v}=10 \mathrm{msec}$. and the results are shown in Fig. 5. Clearly, bipolar input stages feature a better noise performance compared to junction FET-based

$$
\begin{aligned}
& \frac{\overline{u_{o n 1}^{2}}}{\overline{i_{n a}^{2}}} \simeq\left(\frac{-R_{f}\left(1+j \omega R_{s} C_{j}\right)}{1+j \omega\left(R_{f} C_{f}+R_{s} C_{j}\right)-\omega^{2} R_{f} C_{j}\left(R_{s} C_{f}+\frac{\tau_{v}}{A_{o}}\right)}\right)^{2} \\
& \frac{\overline{u_{o n 2}^{2}}}{\overline{\overline{u_{n a}^{2}}}} \simeq\left(\frac{-j \omega R_{f} C_{j}}{1+j \omega\left(R_{f} C_{f}+R_{s} C_{j}\right)-\omega^{2} R_{f} C_{j}\left(R_{s} C_{f}+\frac{\tau_{v}}{A_{o}}\right)}\right)^{2}
\end{aligned}
$$

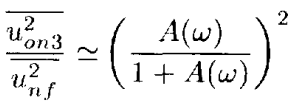




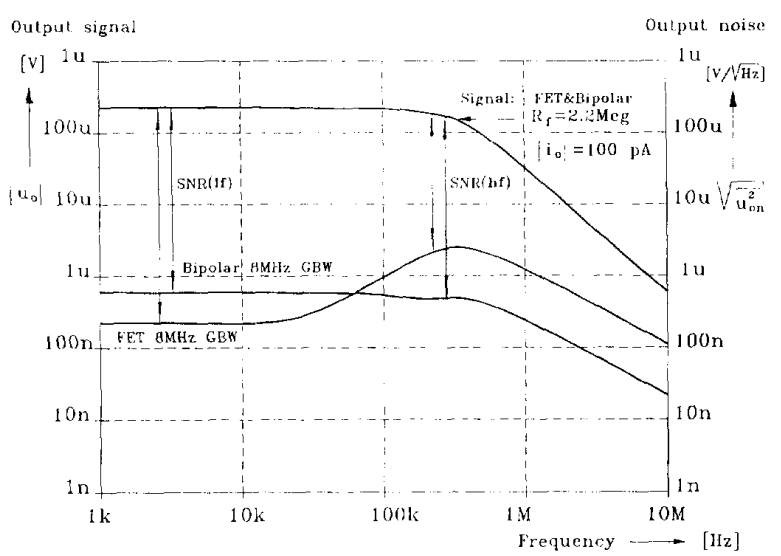

Fig. 5. Detection limit (NEP) and signal-to-noise ratio (SNR) versus frequency for bipolar and FET readout.

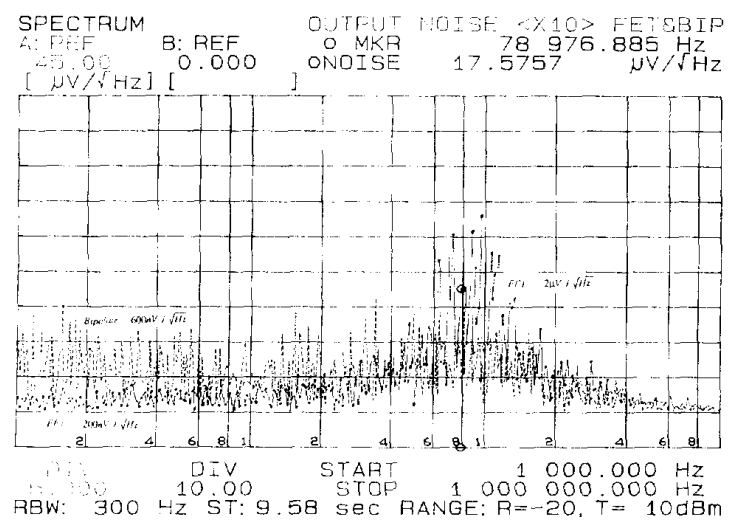

Fig. 6. Measured output spectral noise voltage of both transimpedance amplifiers.

operational amplifiers for frequencies between $80 \mathrm{kHz}$ and $300 \mathrm{kHz}$.

The noise performance has been verified using commercially-available operational amplifiers with either an input stage based on JFET's or bipolar transistors, however both have equal unity gain bandwidth, $f_{T}=8 \mathrm{MHz}$. Fig. 6 shows the measured output noise spectral power in the frequency range of interest. Clearly, the flat response of the transimpedance amplifier is noticeable when using a bipolar operational amplifier when compared to the peaked response of the operational amplifier with a JFET input stage.
A $1 \mathrm{~mm}^{2}$ photodiode has been used with a responsitivity at a $600 \mathrm{~nm}$ wavelength of impinging light at $0.5 \mathrm{~A} / \mathrm{W}$. The noise equivalent power (NEP) of the optical measurements system when operating at $200 \mathrm{kHz}$ and $100 \mathrm{~Hz}$ bandwidth amounts to $14 \mathrm{pW}$ when using a JFET operational amplifier, whereas equal to $5.5 \mathrm{pW}$ in case of a bipolar operational amplifier. This NEP increases from $1.8 \mathrm{pW}$ at $1 \mathrm{kHz}$ to $14 \mathrm{pW}$ at 200 $\mathrm{kHz}$ in case of an operational amplifier with a JFET input stage and remains constant up to frequencies at which the transimpedance drops due to the finite gain-bandwidth of the operational amplifier in the case of bipolar readout.

\section{CONCLUSIONS}

The equivalent input current noise limits the noise performance of a transimpedance amplifier used for photodiode readout only at lower frequencies, whereas the equivalent noise voltage is the limiting factor at high frequencies. Therefore, JFET based readout is used for the readout of conventionally chopped photodiodes. The chopping frequency of such devices was limited to several kiloHertz. The dramatic increase in operating frequency offered by micromechanical choppers would allow operation beyond $1 \mathrm{MHz}$. Bipolar readour circuits have been demonstrated to offer superior noise performance at such a frequency. Nevertheless, the detection limit of optical systems based on mechanical chopping of the impinging light increases with increasing operating frequency, which would make the increase of the chopper frequency undesirable, despite the reduced inertia of integrated micromechanical choppers. However, the potential of a higher operating frequency when considering practical system constraints, such as filter design, nevertheless favours the maximization of operating frequency up to the gain- bandwidth limit of the operational amplifier in many applications. The noise performance is optimized at such elevated frequencies when using bipolar readout circuits.

\section{REFERENCES}

[1] E. H. Nordholt and L. P. de Jong, "The design of extremely low-noise cameratube preamplifiers," IEEE Trans. Instrum. Meas., vol. IM-32, June 1983 .

[2] M. H. El-Diwany, D. J. Roulston, and S. G. Chamberlain, "Design of low-noise bipolar transimpedance amplifiers for optical receivers," IEE Proc. $G$, vol. 128 , no. 6, pp. 299-305. 1981.

[3] C. Linder and N. F. de Rooij, "Investigations on free-standing polysilicon beams in view of their application as transducer," Sensors Actuators, vol. A21-A23, pp. 1053-1059, 1990

[4] W. C. Tang, T. Nguyen, and R. S. Howe, "Electrostatic comb drive of lateral silicon resonators," Sensors Actuators, vol. A2I-A23, pp. $328-331,1990$ 